\title{
ENTOMOFAUNA DO ENTORNO DO RESERVATÓRIO DE ALAGADOS, REGIÃO DOS CAMPOS GERAIS DO PARANÁ, CAPTURADA ATRAVÉS DE ARMADILHA MALAISE
}

\author{
INSECTS AROUND THE RESERVOIR OF ALAGADOS \\ CAPTURED BY MALAISE TRAPS IN THE CAMPOS GERAIS REGION, \\ PARANÁ STATE
}

\author{
Patricia Elizabeth Husch ${ }^{1}$, Julianne Milléo ${ }^{2,}$ Ivana de Freitas Barbola ${ }^{2}$, \\ Jonathan Pena Castro ${ }^{3}$ \\ ${ }^{1}$ Universidade Estadual de Ponta Grossa, Mestrado em Agronomia, Ponta Grossa/PR. \\ E-mail: patihusch@gmail.com. \\ ${ }^{2}$ Universidade Estadual de Ponta Grossa, Departamento de Biologia Geral, Ponta Grossa/ \\ PR.E-mail: jmilleo@hotmail.com, ibarbola@yahoo.com.br; \\ ${ }^{3}$ Universidade Estadual de Ponta Grossa, Mestrado em Biologia Evolutiva, Ponta Grossa/ \\ PR. E-mail: jonathan.penacastro@gmail.com
}

Recebido para publicação em 21/05/2010

Aceito para publicação em 29/06/2010

\section{RESUMO}

O reservatório de Alagados é utilizado para captação de água e abastecimento das cidades de Ponta Grossa, Castro e Carambeí. Apesar da importância para os municípios, a ação antrópica vem causando inúmeras agressões ao reservatório. Tendo em vista a relevância dos insetos como indicadores ambientais, o presente estudo teve como objetivo inventariar, identificar e caracterizar as ordens de insetos encontradas no local. O período de amostragem foi de novembro de 2005 a abril de 2006, em seis pontos de coleta ao longo da margem esquerda do reservatório. $\mathrm{O}$ acesso a esses locais se deu com auxílio de barco e barqueiro cedidos pela SANEPAR. O material recolhido foi triado, identificado, contabilizado, e nos resultados foram avaliados os índices de frequência, constância, abundância e riqueza, considerando-se a influência dos fatores climáticos do período. Foram capturados 1.036 insetos distribuídos em 10 ordens. Sendo Diptera classificada como constante em todos os pontos, esteve em mais de $50 \%$ dos levantamentos e teve maior frequência em cinco dos seis locais amostrados, correspondendo a mais de $68 \%$ do total de insetos coletados. Lepidoptera e Coleoptera aparecem em segundo e quarto lugares quanto à abundância e constantes em todas as amostragens. Hymenoptera, por sua vez, foi a terceira ordem em abundância e constante em quatro dos seis pontos amostrados. A presença significativa dos dípteros, principalmente da família Chironomidae, confirma que em vários pontos do reservatório o ambiente está impactado. Portanto, este trabalho vem contribuir para o conhecimento da diversidade de insetos encontrados no entorno do reservatório de Alagados.

Palavras-chave: Insecta. Biodiversidade. Monitoramento ambiental. 


\begin{abstract}
The Alagados reservoir is used to obtaining and providing water for the cities of Ponta Grossa, Castro and Carambei. Despite the importance for the cities, the anthropic action has been causing numerous damages to the reservoir. Considering the relevance of insects as environmental indicators, the aim of this study was to create an inventory, identify and characterize the insects found in the area. The period of the sampling was from November 2005 to April 2006 in six collecting spots along the left margin of the reservoir. The access to these spots was aided by a boat and a boatman provided by the water company (SANEPAR). The material collected was screened, identified, classified and the results demonstrate the frequency, regularity, quantity and richness indexes taking into consideration the climatic influence of the period of collection. A hundred and thirty-six (1036) insects from 10 orders were captured. The Diptera order was the most frequent order in all the spots; it was present in $50 \%$ of the samples and had a higher frequency in 5 of the 6 sampled areas, which corresponds to more than $68 \%$ of the total of insects collected. Lepidoptera and Coleoptera orders were the second and fourth orders in terms of quantity and regularity in all samples. The Hymenoptera order was the third order in quantity and regularity in 4 of the 6 sampled areas. The significant presence of these diptera, especially the Chironomidae family, confirms that in numerous spots of the reservoir the environment has been affected. This study contributes with knowledge about the diversity of insects found in the Alagados reservoir area.
\end{abstract}

Keywords: Insecta. Biodiversity. Environmental monitoring.

\section{Introdução}

O reservatório de Alagados, situado na divisa dos municípios de Ponta Grossa, Castro e Carambeí, foi construído em 1929 para geração de energia elétrica. É formado pelo barramento do rio Pitangui, com a contribuição do rio Jotuva, aproveitando o desnível existente na entrada do rio Pitangui no segundo planalto. A partir de 1969, passou-se a utilizar o reservatório para captação de água e abastecimento da cidade de Ponta Grossa, respondendo por mais de $38 \%$ do volume de água consumida na cidade. Atualmente, contribui também para o abastecimento de água nos municípios de Castro e Carambeí. (NUCLEAM, 2002).

Apesar da importância do reservatório para os municípios, a ação antrópica vem causando inúmeras agressões ao reservatório: poluição da água pela lixiviação de fertilizantes e de agrotóxicos, contaminação com dejetos humanos e de suínos, desmatamento das margens causando processo erosivo acentuado. A ocupação das margens do reservatório vem acontecendo desde a instalação da barragem e consequente formação do lago. O local de grande beleza cênica tem suas margens ocupadas com diversas construções e utilizadas como área de lazer e suas águas servem para prática de esportes náuticos. Entre as atividades agropecuárias desenvolvidas no entorno destacam-se a criação de suínos, bovinos, ovelhas e aves; além do cultivo de soja, feijão, milho, trigo, aveia e cevada. Existem alguns pontos ativos de exploração de minérios, tais como: saibreiras, cascalheiras e pedreiras. (NUCLEAM, 2002).

Com o intuito de minimizar os impactos ambientais no reservatório de Alagados, o Núcleo de Estudos em Meio Ambiente (NUCLEAM) da Universidade Estadual de Ponta Grossa, em parceria 
com Companhia de Água e Saneamento do Paraná (SANEPAR - Ponta Grossa) vem desenvolvendo estudos multidisciplinares e realizando algumas ações através do Projeto de Preservação da Represa Alagados. Entre os trabalhos desse projeto pode-se destacar o de Barbola et al. (dados não publicados) ${ }^{1}$ que avaliaram diferentes medidas bióticas da comunidade de invertebrados no local.

Alguns organismos, devido às suas características, costumam reagir rapidamente aos impactos e às mudanças ambientais. Os representantes da classe Insecta costumam ser indicadores apropriados para essa finalidade, pois são numerosos e têm grande diversidade, além da capacidade de formar várias gerações em um curto espaço de tempo (SILVEIRA NETO et al., 1995). Segundo Antonini et al. (2003) os insetos possuem diversas características que os tornam indicados para estudos ambientais, tais como: facilidade de amostragem; participam na regulação de populações vegetais e de alguns animais; auxiliam na decomposição da matéria orgânica, na aeração do solo e na ciclagem de nutrientes; participam de diversas interações ecológicas e do fluxo de energia e atuam como polinizadores, dispersores e predadores de sementes, entre outras. Para Freitas et al. (2003), os insetos podem fornecer mais informações que os vertebrados, sendo muito úteis na definição de áreas pequenas e habitats fragmentados ou com longa história de influência antrópica, pois são especialistas em recursos específicos, possuindo fidelidade de micro-habitat e permitindo ações rápidas, como reação a degradação do habitat.

Embora existam alguns trabalhos sobre a entomofauna na região dos Campos Gerais, nenhum trata da diversidade que habita a região do entorno do reservatório de Alagados. Tendo em vista a relevância dos insetos como indicadores ambientais, o presente estudo teve como objetivo inventariar, com uso de armadilha Malaise, identificar e caracterizar qualitativamente e quantitativamente as ordens de insetos encontradas no local. Com esses resultados pretende-se disponibilizar dados para o monitoramento desses bioindicadores.

${ }^{1}$ BARBOLA, I. F.; ANAZAWA, T. M.; MORAES, M. F. P. G.; NASCIMENTO, E. A.; POlEGATTO, C. M.; MillÉO, J.; SCHÜHli, G. S., em estudos sobre a macrofauna de invertebrados das zonas litorâneas e sublitorâneas de um reservatório da bacia do rio Pitangui.

\section{Material e métodos}

\section{1 Área de estudo}

O reservatório de Alagados situa-se numa área de transição entre o Primeiro e o Segundo Planaltos Paranaense, entre as coordenadas $24^{\circ} 52^{\prime}$ a $25^{\circ} 05^{\prime}$ de latitude $\mathrm{S}$ e $49^{\circ} 46^{\prime}$ a $50^{\circ} 06^{\prime}$ de longitude W, abrangendo parte dos municípios de Ponta Grossa, Castro e Carambeí. É uma barragem artificial construída sobre o rio Pitangui, com contribuição do rio Jotuva. Tem extensão de quase $15 \mathrm{~km}$, largura média de $500 \mathrm{~m}$ e profundidade variando em torno de $15 \mathrm{~m}$, próximo à barragem, e de $2 \mathrm{~m}$ a $5 \mathrm{~m}$ no restante da área.

Segundo Cruz (2007) o clima da região é do tipo $\mathrm{Cfb}$, temperado com temperaturas médias abaixo de $18^{\circ} \mathrm{C}$ nos meses mais frios e de $22^{\circ} \mathrm{C}$ nos meses mais quentes; pluviosidade média entre 1.600 $\mathrm{mm}$ e $1.800 \mathrm{~mm}$ anuais, sendo o mês de janeiro o mais chuvoso e agosto o menos chuvoso, sem estação seca definida; umidade relativa média do ar de $75 \%$ a $80 \%$; com ventos suaves e moderados entre $20 \mathrm{~km} / \mathrm{h}$ e $50 \mathrm{~km} / \mathrm{h}$.

Sobre a vegetação na região dos Campos Gerais, Moro e Carmo (2007) comentam que, devido aos afloramentos rochosos e solos dominantemente rasos e pobres, estabeleceu-se uma vegetação predominantemente herbácea, com elementos arbustivos lenhosos. Essa vegetação está frequentemente associada a capões de florestas com araucária (Floresta Ombrófila Mista Montana), a florestas riparias (Floresta Ombrófila Mista Aluvial) ao longo dos cursos d'água e, ocasionalmente, a fragmentos de cerrado (Savana).

\subsection{Confecção das armadilhas}

Para captura dos insetos utilizou-se de armadilhas do tipo Malaise, cujas técnicas de montagem foram adaptadas das descritas em Almeida et al. (1998). Neste trabalho deu-se preferência à utilização de materiais reciclados, sendo assim, potes plásticos de maionese com capacidade de $500 \mathrm{ml}$ foram transformados em recipientes coletores de 
insetos e junto a esses foram acoplados, com Durepoxi ${ }^{\circledR}$, canos de PVC curvilíneos com cerca de duas polegadas de diâmetro para captação dos insetos na extremidade mais alta da armadilha.

\subsection{Pontos de coleta}

Foram selecionados seis pontos de coleta ao longo da margem esquerda do reservatório, que é a menos habitada (Figura 1) e, portanto, menos sujeita à depredação, sendo que a vegetação predominante nos locais constitui-se de fragmentos de Floresta Ombrófila Mista (MORO et al., 2005). Além disso, os pontos 1 e 2 eram próximos a residências; 3 ao lado de área cultivada; 4 e 5 apresentavam a vegetação mais preservada; e o 6 ficava próximo à área de silvicultura. $\mathrm{O}$ acesso aos locais só era possível com uso barco e barqueiros que foram cedidos pela SANEPAR.

\subsection{Amostragem}

O período de amostragem teve início em novembro de 2005 e estendeu-se até abril de 2006, totalizando seis meses de coleta. Após a montagem das oito armadilhas, o recolhimento do conteúdo dos frascos coletores foi programado para ocorrer quinzenalmente. Dois fatores interferiram no andamento do projeto: a) fatores climáticos: período de chuvas constantes e intensas no final de 2005 e período de estiagem, a partir de abril de 2006. Como os pontos de coleta só eram acessíveis com o uso do barco, das 11 amostragens previstas apenas sete realmente foram efetivadas; b) ação antrópica: das oito armadilhas montadas, seis não foram depredadas.

\subsection{Armazenamento e identificação dos insetos coletados}

O material recolhido do frasco coletor das armadilhas foi levado ao laboratório para triagem. Os insetos foram separados, identificados segundo suas ordens (TRIPLEHORN; JOHNSON, 2005) e contabilizados. Após as identificações, e devido ao grande número de dípteros coletados e de sua importância como bioindicadores, esses foram identificados segundo a família. Os exemplares foram conservados em potes com álcool hidratado a $70 \%$ e etiquetados com dados referentes a cada ponto, data, local e nome dos coletores.

\subsection{Catalogação e análise dos resultados}

O levantamento descritivo dos dados utilizou informações provenientes do período e pontos de amostragem, possibilitando a elaboração de um banco de dados através da planilha eletrônica Microsoft Excel e a análise de alguns índices faunísticos, adaptados de Silveira Neto et al. (1976), tais como: Frequência, Constância, Abundância e Riqueza.

A Frequência foi calculada pela soma do número de indivíduos de uma determinada ordem dividida pelo total dos insetos capturados, obtendo-se uma porcentagem para cada ponto. A Constância foi a porcentagem de ocorrência das ordens presentes nos levantamentos executados, calculada pela seguinte fórmula: $\mathrm{C}=\mathrm{P} \times 100 / \mathrm{N}$, sendo $\mathrm{P}$ - número de coletas contendo a ordem; $\mathrm{N}$ - número total de coletas realizadas. $\mathrm{O}$ valor de Abundância corresponde ao número total de insetos capturados, independentemente das ordens às quais eles pertenciam. E a Riqueza avaliou a quantidade de ordens encontradas, sem considerar o número de espécimes capturados em cada uma delas.

Com o intuito de identificar se os fatores climáticos Precipitação Pluviométrica, Temperatura Máxima e Temperatura Mínima variaram significativamente ao longo do período de amostragem foi realizado o teste "t one-sample" (através do software livre PAST). Foi também testado se esses fatores influenciariam na ocorrência dos insetos do entorno de Alagados, através de uma análise de regressão múltipla (utilizando o software livre BioEstat versão 5.0). 


\section{Resultados}

Nos seis meses de coleta, foram capturados 1.036 insetos, distribuídos em 10 ordens. Os resultados foram analisados e encontram-se resumidos na Tabela 1.

Diptera foi classificada como constante em todos os pontos, aparecendo em mais de $50 \%$ dos levantamentos efetuados. Essa ordem apresentou também maior frequência em cinco dos seis locais amostrados, correspondendo a mais de $68 \%$ do total de insetos coletados. Lepidoptera e Coleoptera, apesar de menos frequentes, aparecem em segundo e quarto lugares quanto à abundância e foram classificados como constantes em todas as amostragens realizadas. Hymenoptera aparece como a terceira ordem em abundância e constante em quatro dos seis pontos amostrados. Blattodea, Psocoptera, Hemiptera, Neuroptera, Isoptera e Orthoptera foram pouco frequentes e classificadas como acessórias ou acidentais na constância das amostragens.

Analisando-se o item abundância nos locais de coleta, a sequência em ordem decrescente foi: ponto $2(28,0 \%)$, ponto $5(20,8 \%)$, ponto $4(20,7 \%)$, ponto $3(17,3 \%)$, ponto $6(7,0 \%)$ e ponto $1(6,3 \%)$. Das 10 ordens coletadas, apenas quatro estavam presentes em todos os locais: Diptera, Lepidoptera, Hymenoptera e Coleoptera (Tabela 1). Em relação à riqueza, a disposição decrescente foi: pontos 3 e 6 (sete ordens), pontos 2, 4 e 5 (seis ordens) e ponto 1 (cinco ordens). Os pontos classificados como mais abundantes ( 2 e 5) não foram os que apresentaram maior riqueza de ordens ( 3 e 6 ).

As flutuações de abundância e riqueza de táxons, assim como de alguns parâmetros climáticos estão sumarizados na Figura 3. A pluviosidade variou significativamente durante o período amostrado $(\mathrm{t}=4,4017 ; \mathrm{p}=0,0035)$, o mesmo tendo sido observado em relação às temperaturas mínimas $(\mathrm{t}$ $=17,6092 ; \mathrm{p}<0,0001)$ e máximas $(\mathrm{t}=36,4198 ; \mathrm{p}$ $<0,0001)$.

Resultados de regressão múltipla indicam que a riqueza dos macroinvertebrados (número de táxons) no entorno de Alagados foi influenciada mais fortemente pela temperatura máxima do ar (b $=1,0973 ; \mathrm{p}=0,0485)$ e fracamente influenciada pela temperatura mínima $(b=-0,5696 ; p=0,3692)$ e pluviosidade $(b=-0,013 ; p=0,3987)$, sendo o modelo de regressão significativo ao nível de $\mathrm{p}=$ 0,0621 e $\mathrm{R}^{2}$ ajustado $=0,7757$. Para a abundância de indivíduos, o modelo de regressão $\left(\mathrm{R}^{2}\right.$ ajustado $=0,3135 ; \mathrm{p}=0,2081)$ foi fracamente definido pelos parâmetros abióticos analisados $(b=129,8419$ e $p$ $=0,1749$ para temperatura máxima; $b=-232,6264$ e $\mathrm{p}=0,2020$ para temperatura mínima; $\mathrm{b}=2,6837$ e $\mathrm{p}=0,4604$ para pluviosidade).

\section{Discussão}

O desenvolvimento larval aquático de muitas famílias de Diptera justifica a presença numerosa nos locais de coleta ao longo da margem do reservatório. As três famílias mais representativas foram: Chironomidae, Tabanidae (cujas larvas são aquáticas) e Asilidae (com larvas que se desenvolvem no solo). Barbola et al. (dados não publicados) também capturaram um grande número de larvas de dípteros ao analisar a diversidade da macrofauna de invertebrados aquáticos no reservatório de Alagados, sendo que a família Chironomidae foi considerada dominante e constante nas coletas, constituindo $68,0 \%$ da comunidade de macroinvertebrados no local. A predominância de dípteros, principalmente da família Chironomidae, considerados bioindicadores de ambientes eutrofizados - isto é, as larvas desenvolvem-se preferencialmente em águas com baixa concentração de oxigênio e alta quantidade de matéria orgânica -, indicam ecossistema impactado, principalmente na altura dos pontos 2, 3, 4 e 5 .

O maior número de lepidópteros (borboletas e mariposas) foi observado em pontos de vegetação parcialmente preservada; esses insetos são citados em Freitas et al. (2003) como bons indicadores ambientais. Já a presença dos coleópteros justifica-se por tratar-se da ordem mais numerosa de insetos, representando $40 \%$ do total. As ordens Blattodea, Psocoptera, Hemiptera, Neuroptera, Isoptera e Orthoptera foram pouco representativas e não apresentam espécies descritas na literatura como indicadoras de qualidade ambiental. Em relação à abundancia de espécies constatou-se um valor alto 
nos locais menos sujeitos à ação antrópica (pontos 2, 5 e 4).

Embora não tenha sido confirmada estatisticamente, é possível que as temperaturas mínimas mais baixas e a quantidade de chuvas nos meses de fevereiro e março, além de outros fatores do ambiente, como umidade relativa, por exemplo, tenham contribuído para a diminuição na abundância de insetos nesse período. Marinoni e Dutra (1991) descrevem que a queda de temperatura nos meses de verão, acompanhada do aumento na precipitação podem determinar uma diminuição na densidade de insetos na região.

De um modo geral, os resultados obtidos guardam semelhanças com outros estudos realizados na região, por Marinoni e Dutra (1991), no Parque Estadual de Vila Velha (PEVV), e por Nascimento et al. (2002), em um fragmento de Floresta Ombrófila Mista, no Parque Nacional dos Campos Gerais (PNCG). Nesses inventários, em que os autores também utilizaram armadilhas tipo Malaise, Diptera aparece como a ordem mais abundante, representando $87,1 \%$ da amostra do PEVV e $70,6 \%$ do PNCG. Seguem Hymenoptera (5,4\%), Lepidoptera $(2,7 \%)$ e Coleoptera (2,0\%) no PEVV, enquanto no PNCG alguns valores se invertem, sendo que Hymenoptera se mantém como a segunda ordem mais abundante $(11,5 \%)$, seguida de Coleoptera $(6,1 \%)$, Hemiptera $(5,8 \%)$ e Lepidoptera $(4,4 \%)$.

Dutra e Marinoni (1994), na Ilha do Mel, Paraná, e Santos et al. (2002), no Jardim Zoológico da cidade de Goiânia, também capturaram mais dípteros, lepidópteros himenópteros, coleópteros e hemípteros com armadilhas Malaise. Esse fato deve-se, em parte, à seletividade da armadilha, que captura principalmente insetos com voo baixo, diurnos ou noturnos, em áreas florestadas e, em parte, às características dessas ordens consideradas megadiversas.

\section{Conclusões}

Este trabalho vem contribuir para o conhecimento da diversidade de insetos encontrados no entorno do reservatório de Alagados. A presença significativa dos dípteros, principalmente da família Chiromonidae, confirma que em vários pontos do reservatório o ambiente está impactado.

\section{Agradecimentos}

Agradecemos ao PIBIC/CNPq/Fundação Araucária pela concessão da bolsa de iniciação científica; à Companhia de Água e Saneamento do Paraná (SANEPAR - Ponta Grossa) pelo apoio nos trabalhos de campo.

\section{REFERÊNCIAS}

ALMEIDA, L. M. de; RIBEIRO-COSTA, C. S.; MARINONI, L. Manual de coleta, conservação, montagem e identificação de insetos. Ribeirão Preto: Holos, 1998. 88p.

ANTONINI, A.; ACCACIO, G. M.; BRANT, A.; CABRAL, B. C.; FONTENELLE, J. C. R.; NASCIMENTO, M. T.; THOMAZINI, A. P. B. W.; THOMAZINI, M. J. 2003. Insetos. In: RAMBALDI, D. M.; OLIVEIRA, D. A. S. Fragmentação de ecossistemas: causas, efeitos sobre a biodiversidade e recomendações de políticas públicas. Brasília: MMA./SBF, p. 239-273, 2003.

CRUZ, G. C. F. Alguns aspectos do clima dos Campos Gerais. In: MELO, M. S.; MORO, R. S.; GUIMARÃES, G. B. (Eds.). Patrimônio Natural dos Campos Gerais do Paraná. Ponta Grossa: Editora UEPG, 2007. p. 59-72.

DUTRA, R. R. C.; MARINONI, R. C. Insetos capturados com armadilha Malaise na Ilha do Mel, Baía de Paranaguá, Paraná, Brasil. II Tabanidae (Diptera). Ver. Bras. Zool., v. 11, n. 2, p. 247-256, 1994.

FREITAS, A. V. L.; FRANCINI, R. B.; BROWN Jr. K. S. Insetos como indicadores ambientais. In: MÉTODOS de estudos em biologia da conservação e manejo da vida. Curitiba PR: Editora UFPR, Fundação O Boticário de Proteção à Natureza, 2003. $667 \mathrm{p}$.

MARINONI, R. C.; DUTRA R. R. C. Levantamento da fauna entomológica no Estado do Paraná. I- Introdução. Situações climática e florística de oito pontos de coleta. Dados faunísticos de Agosto de 1986 a Julho de 1987. Rev. Bras. Zool., v. 8, p. 31-73, 1993.

MORO, J. C.; COSTA, E. T. V.; MILANESE, S.; MORO, R. S. Comparação da cobertura vegetal nas áreas de preservação permanente na Represa de Alagados (PR), de 1980 a 2001. Publ. UEPG Ci. Biol. Saúde, Ponta Grossa, v. 11, n. 2, p. 13-20, jun. 2005. 
MORO, R. S.; CARMO, M. R. B. A vegetação campestre nos Campos Gerais In: MELO, M. S.; MORO, R. S.; GUIMARÃES, G.B. (Eds.). Patrimônio Natural dos Campos Gerais do Paraná. Ponta Grossa: Editora UEPG, p. 93-98, 2007.

NASCIMENTO, E. A.; MICHELI, A.; BARBOLA, I. F. Inventário da entomofauna dos sítios naturais dos Campos Gerais do Paraná, Brasil. In: CONGRESSO BRASILEIRO DE ZOOLOGIA, 24. Itajaí, 2002.

NÚCLEO DE ESTUDOS EM MEIO AMBIENTE (NUCLE$\mathrm{AM}$ ). Bacia hidrográfica do manacial alagados. Relatório técnico. Ponta Grossa: Universidade Estadual de Ponta Grossa, 2002. 145p.

SANTOS, B. B.; SILVA, S. S. Ordens de insetos coletados com armadilha Malaise no Parque Zoológico de Goiânia, Goiás, Brasil. In: CONGRESSO BRASILEIRO DE ZOOLOGIA, 24. Itajaí, SC. Resumos... v.24, p.165, 2002.

SILVEIRA NETO, S.; NAKANO, O.; BARBIN, D.; VILLA NOVA, N. A. Manual de ecologia dos insetos. Piracicaba: Editora Agronômica Ceres, 1976. 419p.

SILVEIRA NETO, S.; MONTEIRO, R. C.; ZUCCHI, R. A.; MORAES, R. C. B. Uso da análise faunística de insetos na avaliação do impacto ambiental. Sci. Agric., Piracicaba, v.52, n.1, p.9-15, 1995.

TRIPLEHORN, C. A.; JOHNSON, N. F. Borror and Delong's introduction to the study of insects. 7. ed. Belmont: Thonson Brooks/Cole, 2005. 864p.
Figura 2 - Variação mensal do número de táxons (\% riqueza), de indivíduos (abund.), pluviosidade (pluv.) e temperaturas máximas e mínimas $\left(\mathrm{T}^{\circ} \mathrm{C}\right.$ max. e $\mathrm{T}^{\circ} \mathrm{C}$ min.)

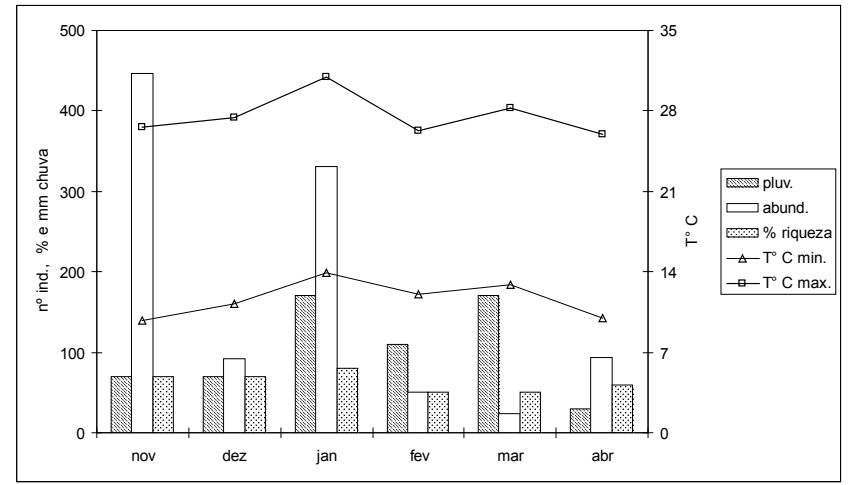

Fonte das temperaturas e pluviosidade: SIMEPAR

\section{Anexos}

Figura 1 - Reservatório de Alagados com indicação dos pontos de coleta

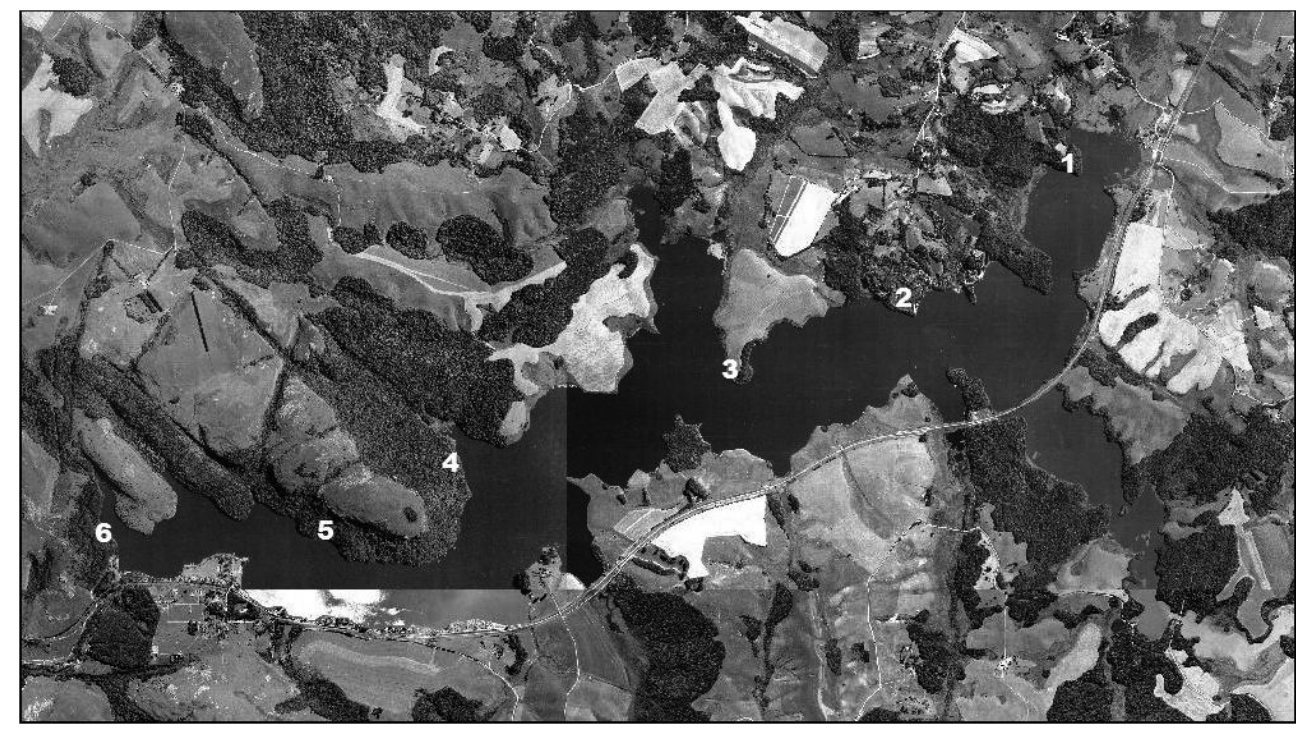

Fonte: Arquivo NUCLEAM. 
Tabela 1 - Ordens de Insecta amostradas em seis pontos de coleta no reservatório de Alagados (Ponta Grossa-PR.), de nov/05 a abr/06

\begin{tabular}{|c|c|c|c|c|c|c|c|c|c|c|c|c|c|c|c|c|c|c|c|c|}
\hline \multirow{2}{*}{ ORDENS } & \multicolumn{3}{|c|}{ PONTO 1} & \multicolumn{3}{|c|}{ PONTO 2} & \multicolumn{3}{|c|}{ PONTO 3} & \multicolumn{3}{|c|}{ PONTO 4} & \multicolumn{3}{|c|}{ PONTO 5} & \multicolumn{3}{|c|}{ PONTO 6} & \multirow[t]{2}{*}{ TOTAL } & \multirow[t]{2}{*}{$\%$} \\
\hline & $\mathbf{N}$ & $\mathbf{F}$ & $\mathbf{C}$ & $\mathbf{N}$ & $\mathbf{F}$ & $\mathbf{C}$ & $\mathbf{N}$ & $\mathbf{F}$ & $\mathbf{C}$ & $\mathbf{N}$ & $\mathbf{F}$ & $\mathbf{C}$ & $\mathbf{N}$ & $\mathbf{F}$ & $\mathbf{C}$ & $\mathbf{N}$ & $\mathbf{F}$ & $\mathbf{C}$ & & \\
\hline Diptera & 40 & $f$ & $\mathrm{X}$ & 218 & $\mathrm{f}$ & $\mathrm{x}$ & 126 & $\mathrm{f}$ & $\mathrm{X}$ & 163 & $\mathrm{f}$ & $x$ & 122 & $\mathrm{I}$ & $x$ & 36 & $\mathrm{pf}$ & $\mathrm{x}$ & 705 & 68,05 \\
\hline Lepidoptera & 11 & $\mathrm{pf}$ & $\mathrm{X}$ & 21 & $\mathrm{pf}$ & $\mathrm{x}$ & 10 & $\mathrm{pf}$ & $\mathrm{X}$ & 24 & $\mathrm{pf}$ & $\mathrm{x}$ & 35 & $\mathrm{pf}$ & $\mathrm{x}$ & 10 & $\mathrm{pf}$ & $\mathrm{x}$ & 111 & 10,71 \\
\hline Hymenoptera & 5 & $\mathrm{pf}$ & $\mathrm{X}$ & 17 & $\mathrm{pf}$ & $\mathrm{x}$ & 24 & $\mathrm{pf}$ & $\mathrm{X}$ & 14 & $\mathrm{pf}$ & $\mathrm{y}$ & 26 & $\mathrm{pf}$ & $\mathrm{x}$ & 11 & $\mathrm{pf}$ & $\mathrm{y}$ & 97 & 9,36 \\
\hline Coleoptera & 7 & $\mathrm{pf}$ & $\mathrm{X}$ & 31 & $\mathrm{pf}$ & $\mathrm{x}$ & 9 & $\mathrm{pf}$ & $\mathrm{X}$ & 8 & $\mathrm{pf}$ & $\mathrm{x}$ & 29 & $\mathrm{pf}$ & $\mathrm{x}$ & 12 & $\mathrm{pf}$ & $\mathrm{x}$ & 96 & 9,27 \\
\hline Blattodea & 2 & $\mathrm{pf}$ & $\mathrm{Y}$ & 0 & - & - & 0 & - & - & 4 & $\mathrm{pf}$ & $\mathrm{z}$ & 2 & $\mathrm{pf}$ & $\mathrm{y}$ & 1 & $\mathrm{pf}$ & $\mathrm{z}$ & 9 & 0,87 \\
\hline Psocoptera & 0 & - & - & 0 & - & - & 7 & $\mathrm{pf}$ & $\mathrm{X}$ & 0 & - & - & 0 & - & - & 0 & - & - & 7 & 0,68 \\
\hline Hemiptera & 0 & - & & 2 & $\mathrm{pf}$ & $\mathrm{y}$ & 1 & $\mathrm{pf}$ & $\mathrm{Z}$ & 1 & $\mathrm{pf}$ & $\mathrm{z}$ & 1 & $\mathrm{pf}$ & $\mathrm{z}$ & 1 & $\mathrm{pf}$ & $\mathrm{z}$ & 6 & 0,58 \\
\hline Neuroptera & 0 & - & - & 0 & - & - & 0 & - & - & 0 & - & - & 0 & - & - & 2 & $\mathrm{pf}$ & $\mathrm{y}$ & 2 & 0,19 \\
\hline Isoptera & 0 & - & - & 0 & - & - & 2 & $\mathrm{pf}$ & $\mathrm{Z}$ & 0 & - & - & 0 & - & - & 0 & - & - & 2 & 0,19 \\
\hline Orthoptera & 0 & - & - & 1 & $\mathrm{pf}$ & $\mathrm{z}$ & 0 & - & - & 0 & - & - & 0 & - & - & 0 & - & - & 1 & 0,1 \\
\hline TOTAL & 65 & & & 290 & & & 179 & & & 214 & & & 215 & & & 73 & & & 1036 & 100,0 \\
\hline
\end{tabular}

Nota: N: número total de insetos coletados; F: frequência (onde Frequente [f] - ordem cuja porcentagem se manteve acima da média; pouco frequente [pf] - ordem cuja porcentagem se manteve abaixo da média); C: constância (onde ordens constantes [x] - presentes em mais de 50\% das coletas; ordens acessórias [y] - presentes entre 25 e $50 \%$ das coletas; e ordens acidentais $[\mathrm{z}]$ - presentes em menos de $25 \%$ das coletas). 